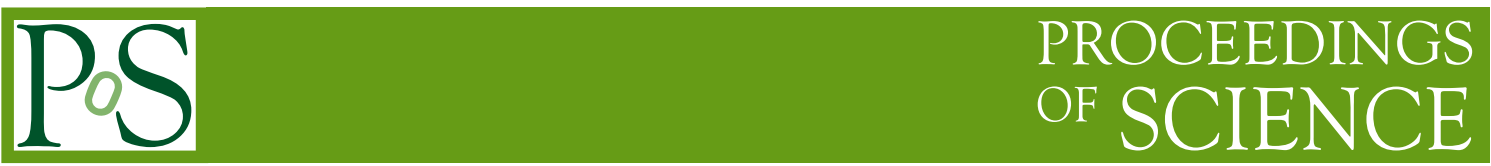

\title{
Recent $\mathrm{H1}$ results on diffraction
}

\section{Sergey Levonian ${ }^{* \dagger}$}

DESY, Notkestraße 85, 22607 Hamburg, Germany

E-mail: levonian@mail.desy.de

Latest results from the $\mathrm{H} 1$ experiment on diffractive processes at HERA are presented. They cover inclusive measurements of diffractive deep inelastic scattering, as well as diffractive dijet production both in DIS and photoproduction regimes. Inclusive cross sections are measured based on the large statistics sample selected by the Large Rapidity Gap technique. Dijet final states are studied by using diffractive samples with tagged leading proton.

Experimental data are compared to several theoretical models and to NLO QCD calculations using diffractive parton densities. Factorisation properties of diffractive $e p$ scattering are tested.

The European Physical Society Conference on High Energy Physics

18-24 July, 2013

Stockholm, Sweden

*Speaker.

†n behalf of the H1 Collaboration 


\section{Introduction}

Diffractive processes have been extensively studied in deep-inelastic ep scattering (DIS) at the HERA collider. Such reactions, $e p \rightarrow e X p$, are characterised by the presence of a leading proton in the final state carrying most of the proton beam energy, and a large rapidity gap (LRG) separating it from the rest of the hadronic system, $X$.

Both short distance and long distance physics contribute to diffractive DIS at essential level. Such a complicated interplay between soft and hard phenomena makes theoretically challenging to understand diffraction at HERA at quantitative level. In 'hadronic language' diffraction is best described in the framework of Regge formalism as a $t$-channel exchange of a leading trajectory with the vacuum quantum numbers, named Pomeron. Specific interest to diffraction as a "physics of Pomeron' is related to the fact that the Pomeron exchange asymptotically dominates over all other contributions to the scattering amplitude, and thus represents the essence of strong interactions in high energy limit. Translating to the modern partonic language this reveals that colourless exchange is important in low- $x$ regime (which is high energy limit of QCD) where gluons expect to dominate.

One of the central problems in hard diffraction is the question of $Q C D$ factorisation, i.e. the question whether it is possible to describe diffractive cross section by a convolution of nonperturbative diffractive parton distribution functions (DPDFs) with universal partonic cross sections. For diffractive DIS QCD collinear factorisation theorem has been proven by Collins [1] while it fails for hadron induced reactions, as measured e.g. at the Tevatron [2]. In addition it is important to test a conjecture of Regge factorisation which assumes that the diffractive cross section can be expressed as a product of Pomeron flux and its structure function.

In this paper we first present final H1 measurement of inclusive diffraction in DIS, based on high statistics LRG sample [3], and then describe diffractive dijet production with tagged proton in DIS regime [4] and in photoproduction[5]. Both hard QCD and Regge factorisation hypotheses are confronted with these data.

\section{Inclusive Diffraction in DIS}

A new measurement [3] of the diffractive neutral current DIS cross section using $375 \mathrm{pb}^{-1}$ data sample recorded with the H1 detector in the years 1999-2000 and 2004-2007 were combined with previously published H1 LRG data [6] of $74 \mathrm{pb}^{-1}$ taken in 1997 and 1999-2000. This provided a final single set of diffractive cross sections covering wide range of photon virtualities, $Q^{2}$ from 3 to $1600 \mathrm{GeV}^{2}$ and diffractive variable $0.0017 \leq \beta=x / x_{\mathbb{P}} \leq 0.8$ in five fixed values of $x_{\mathbb{P}}$ from the interval $0.0003 \leq x_{\mathbb{P}} \leq 0.03$. Here $x_{\mathbb{P}}$ is the fractional momentum loss of the incident proton and $x$ is usual Bjorken variable. The new combined data have a total uncertainty between $4 \%$ and 7\%. The results are corrected to the region $M_{Y}<1.6 \mathrm{GeV}$, and $|t| \leq 1 \mathrm{GeV}^{2}$ and hence contain in addition to elastic diffraction some admixture of low mass proton dissociation. The fraction of $p$-dissociation was estimated by comparing these LRG data with FPS measurements [7] in which outgoing proton is directly tagged in Roman Pots $\left(M_{Y}=M_{p}\right)$ :

$$
\left.\frac{\sigma\left(M_{Y}<1.6 \mathrm{GeV}\right)}{\sigma(Y=p)}=1.203 \pm 0.019(\text { exp. }) \pm 0.087 \text { (norm. }\right)
$$



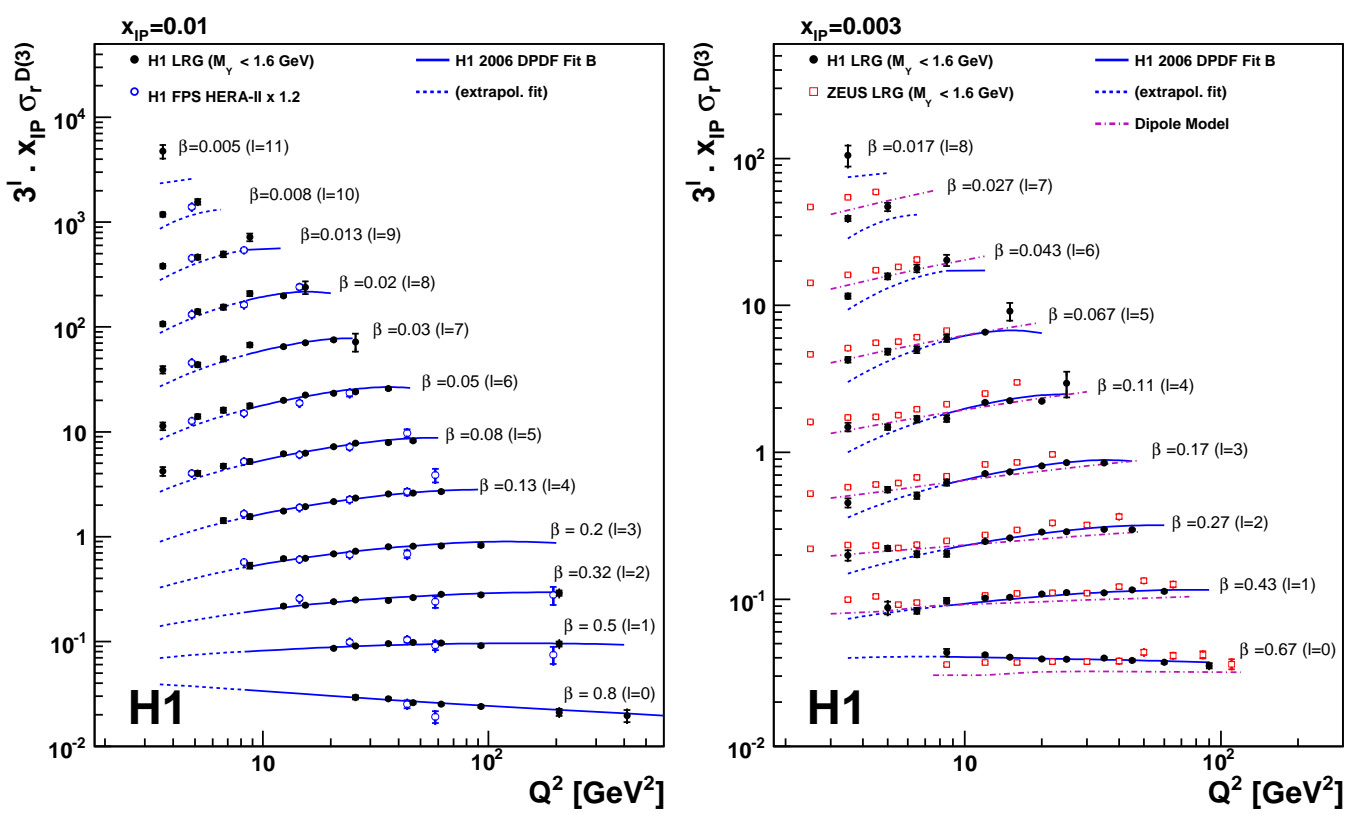

Figure 1: The $Q^{2}$ dependence of the reduced diffractive cross section from combined H1 LRG data, multiplied by $x_{\mathbb{P}}$, in two $x_{\mathbb{P}}$ bins. New H1 data are compared to H1 FPS (left) and to ZEUS LRG (right) measurements. The overall normalisation uncertainties of $\mathrm{H} 1$ and ZEUS data are not shown. Predictions from the H1 DPDF 2006 Fit B are extrapolated below $Q^{2}=8.5 \mathrm{GeV}^{2}$.

In figure 1 new LRG cross sections are compared with H1 FPS measurement and with recent ZEUS results [8]. They are in fair agreement, up to the global normalisation factor $(\sim 10 \%$ between H1 and ZEUS LRG cross sections). The data are also compared to two different QCD models. Figure 1a shows resolved Pomeron model predictions based the H1 2006 DPDF Fit B [6], which assumes proton vertex factorisation. In figure $1 \mathrm{~b}$ the data are compared to the dipole model [9]. In the low $Q^{2}$ range, for $Q^{2}<8.5 \mathrm{GeV}^{2}$, the dipole model, which includes saturation effects, seems to better describe the data, whereas for larger $\beta$ it tends to underestimate the measured cross section.

The diffractive structure function $F_{2}^{D(3)}$, obtained from the reduced cross sections by correcting for the small $F_{L}^{D(3)}$ contribution, can also be interpreted in the framework of Regge phenomenology as a sum of two factorised contributions, corresponding to the leading Pomeron and sub-leading Reggeon terms. Intercept $\alpha_{\mathbb{P}}(0)$ of the linear Pomeron trajectory, $\alpha_{\mathbb{P}}(t)=\alpha_{\mathbb{P}}(0)+\alpha_{\mathbb{P}}^{\prime} \cdot t$, has been extracted by fitting $x_{\mathbb{P}}$ dependencies of $F_{2}^{D(3)}\left(x_{\mathbb{P}}, Q^{2}, \beta\right)$ in several $Q^{2}$ bins independently, while taking $\alpha_{I P}^{\prime}=0.04_{-0.06}^{+0.08} \mathrm{GeV}^{2}$ from previous $\mathrm{H} 1$ measurement [7]. As illustrated by figure 2 no significant $Q^{2}$ dependence of the Pomeron intercept was observed, which supports the proton vertex factorisation hypothesis. The average value is found to be

$$
\alpha_{\mathbb{P}}(0)=1.113 \pm 0.002(\text { exp. })_{-0.015}^{+0.029}(\text { model })
$$

It is interesting to note, that while $\alpha_{\mathbb{P}}(0)$ is close to the 'soft Pomeron' intercept [10], the value of the slope $\alpha_{\mathbb{P}}^{\prime}$ is characteristic to 'hard Pomeron' (no or very small shrinkage). This indicates a complicated interplay of soft and hard phenomena in diffractive DIS. 


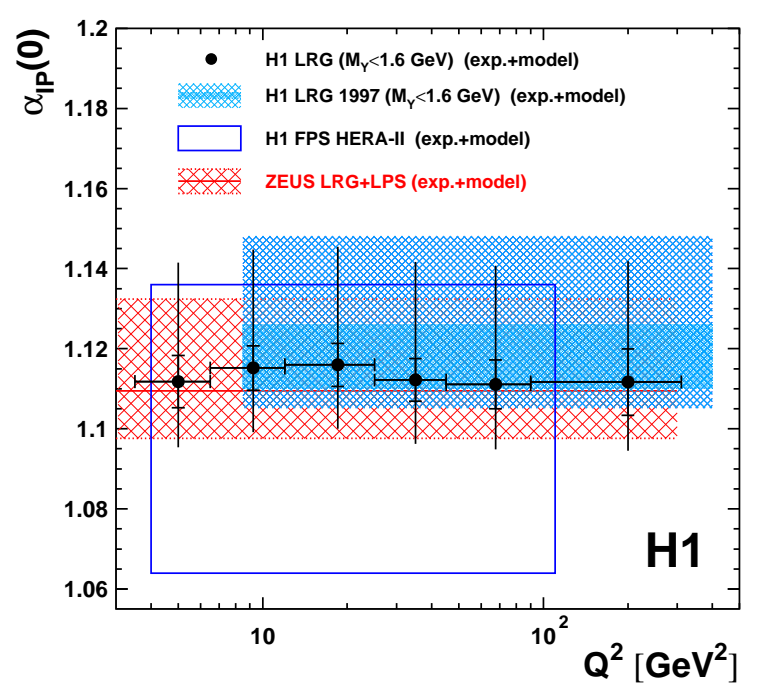

Figure 2: Pomeron intercept values obtained from Regge fits in different $Q^{2}$ bins (dots). The inner error bars represent the statistical and systematic errors added in quadrature and the outer error bars include model uncertainties in addition. Previous determinations of the Pomeron intercept [6-8] are also displayed for comparison. For these previous results the bands or boxes represent the combination of experimental and model uncertainties, always dominated by the model error.

\section{Diffractive dijets and QCD factorisation}

Dijet final states in diffraction at HERA are especially interesting as they directly constrain the diffractive gluon density. Moreover, by investigating dijet production at different $Q^{2}$ one can check QCD factorisation and eventually its breaking in more detail. Namely, one could expect to observe different behaviour of so called gap survival probability in direct ( $x_{\gamma}=1$, DIS-like) and resolved $\left(x_{\gamma}<1, h\right.$-like) photoproduction processes, where $x_{\gamma}$ is a fraction of the photon four-momentum participating in hard subprocess.

New $\mathrm{H} 1$ measurements of the diffractive dijet production with tagged leading proton are available both in DIS [4] and photoproduction [5] regimes. In DIS case elastically scattered proton is detected in FPS stations at $z=61$ and $80 \mathrm{~m}$ from the interaction region, covering kinematic region $x_{\mathbb{P}}<0.1$ and $0.1<|t|<0.7 \mathrm{GeV}^{2}$, while in photoproduction new VFPS $(z=220 \mathrm{~m})$ is used with acceptance of $0.010<x_{\mathbb{P}}<0.024$ and $|t|<0.6 \mathrm{GeV}^{2}$. In both analyses unfolding procedure is applied to account for migration and detector resolution effects. Jets are reconstructed by the longitudinally invariant $k_{T}$-algorithm with jet radius of 1.0.

In DIS sample $\left(4<Q^{2}<110 \mathrm{GeV}^{2}\right)$ dijets are measured for two distinct event topologies. For the 'two central jets' topology (581 events) jets with transverse momentum in the $\gamma^{*} p$ centre of mass system $P_{T, 1}^{*}>5 \mathrm{GeV}$ and $P_{T, 2}^{*}>4 \mathrm{GeV}$ are required in the pseudorapidity range $-1<$ $\eta_{1,2}<2.5$ in the laboratory frame. For the 'one central + one forward jet' topology (309 events) which is motivated by the study of diffractive DIS processes in a phase space where deviations from DGLAP parton evolution may be present, jet selection is as follows. At least one central jet with $-1<\eta_{c}<2.5$ and one forward jet with $1<\eta_{f}<2.8$, where $\eta_{f}>\eta_{c}$, are required with $P_{T}^{*}>3.5 \mathrm{GeV}$. In addition, the invariant mass of the central-forward jet system is required to be larger than $12 \mathrm{GeV}$ to avoid the phase space region in which NLO QCD calculations are unreliable.

The measured cross sections are extrapolated to the region $|t|<1.0 \mathrm{GeV}^{2}$ for easier comparisons with LRG measurements and DPDF fits. Examples of differential cross sections for both event topologies are shown in figure 3. They are fairly well described by NLO QCD calculations using NLOJET++ program [11] and two different H1 DPDF parametrisations. This supports QCD 

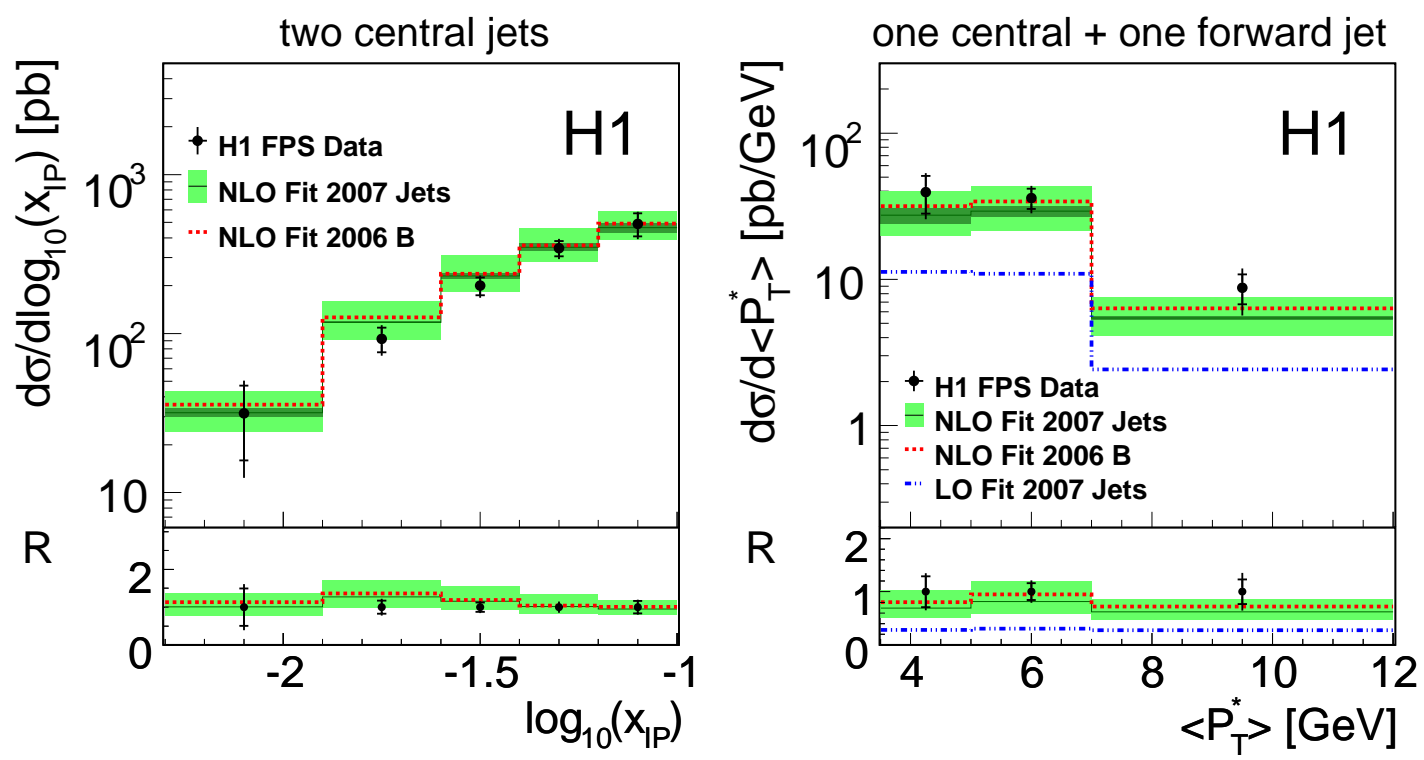

Figure 3: The differential cross section for diffractive dijet production in DIS regime for different jet topologies: two central jets as a function of $\log _{10}\left(x_{\mathbb{P}}\right)$ (left) and one central and one forward jet as a function of $\left\langle P_{T}^{*}\right\rangle$ (right). The inner error bars represent the statistical errors. The outer error bars indicate the statistical and systematic errors added in quadrature. The measured cross sections are compared to NLO QCD predictions based on different DPDF sets. $R$ denotes the ratio of the measured cross sections and QCD predictions to the nominal values of the measured cross sections. The global normalisation error of $7.0 \%(6.2 \%)$ for two central jets (one central and one forward jet resp.) is not shown.
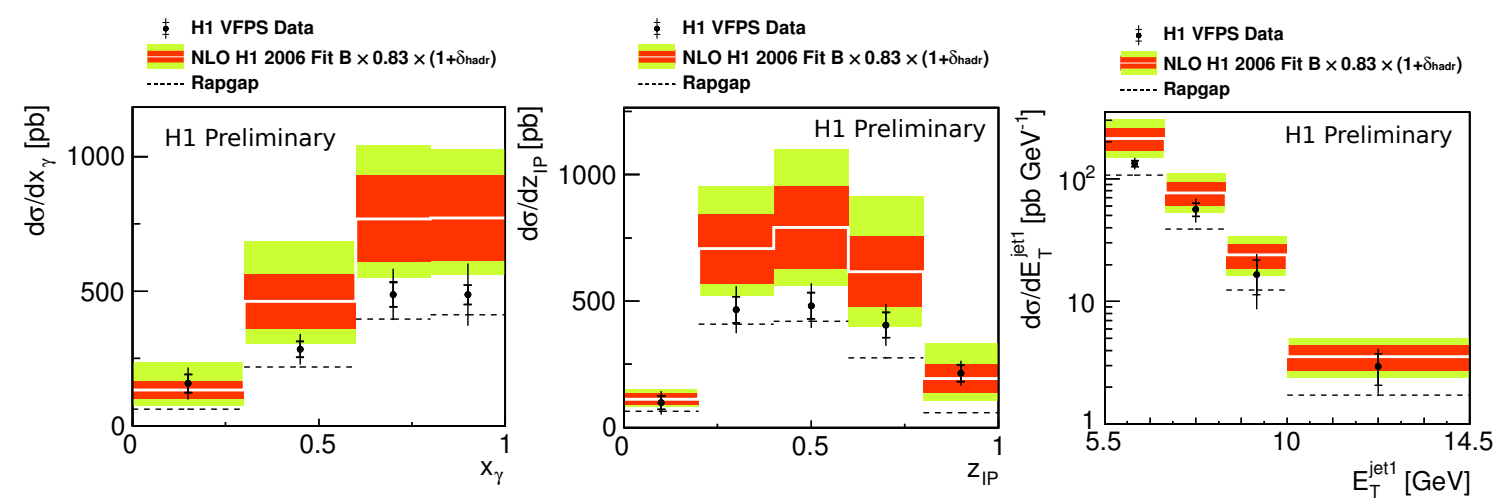

Figure 4: The differential cross section for diffractive dijet photoproduction shown as a function of $x_{\gamma}, z_{\mathbb{P}}$ and $E_{T}^{\text {jet } 1}$. The inner and the outer error bars represent the statistical and total errors respectively. NLO QCD predictions based on the DPDF set H1 2006 Fit B, corrected to the level of stable hadrons, are shown as a white line. The red band indicates the DPDFs uncertainties and light green band indicates the DPDFs and scale uncertainties added in quadrature. LO QCD predictions from Rapgap MC based on the same DPDF set are shown as a dashed black line. 
factorisation and the universality of DPDFs in DIS regime. No evidence of the deviations from DGLAP is found.

For untagged photoproduction analysis $\left(Q^{2}<2 \mathrm{GeV}^{2}\right)$ at least 2 jets with $E_{T}^{\text {jet1 }}>5.5 \mathrm{GeV}$ and $E_{T}^{\mathrm{jet} 2}>4 \mathrm{GeV}$ are required, both in $-1<\eta^{\text {jet1,2 }}<2.5$ range. This gives a sample of 4800 dijet events. Results are compared to the LO Monte Carlo Rapgap [12] and to NLO QCD calculations [13] adapted for diffractive applications. NLO QCD predictions are scaled down by a factor 0.83 in order to correct for proton dissociation fraction in H1 DPDFs extracted from LRG data (see section 2).

The measured differential cross sections as a function of $x_{\gamma}, z_{\mathbb{P}}$ and $E_{T}^{\mathrm{jet} 1}$, where $z_{\mathbb{P}}$ is the Pomeron momentum fraction entering to the hard subprocess, are shown in figure 4. LO MC underestimates the data. NLQ QCD describes the shapes of all distributions fairly well, but overestimates the absolute cross section by a global, $x_{\gamma}$ independent factor, indicating factorisation breaking in diffractive photoproduction. The gap survival probability is measured to be

$$
\sigma_{D A T A} / \sigma_{N L O}=0.67 \pm 0.04(\text { stat }) \pm 0.09 \text { (sys) } \pm 0.20 \text { (scale) } \pm 0.14(\mathrm{PDF})
$$

in agreement with previous H1 measurement [14] using tagged photoproduction and LRG method. This conclusion is somewhat different from the one obtained by ZEUS [15] although at the current level of precision the results agree within large model uncertainty. Also, some weak $E_{T}$ dependence of suppression factor cannot be excluded. In order to sharpen the conclusion the dominant uncertainties of the measurement must be reduced significantly. This could be achieved by measuring double ratio of $\sigma_{D A T A} / \sigma_{N L O}$ in photoproduction and DIS in otherwise the same phase space.

\section{Summary}

Precision measurement is now available, representing a final H1 word on inclusive LRG cross sections in DIS based on full HERA data. These data provide new constraints to QCD models and support proton vertex factorisation hypothesis. By comparison with proton-tagged cross section, a $20 \%$ contribution of proton dissociation is found to be present in H1 LRG data.

Diffractive dijet production is studied both in DIS and photoproduction regimes. In DIS case both shapes of the distributions and the cross section value are well described by NLO QCD calculations, thus supporting QCD factorisation. In photoproduction regime the measured cross sections are smaller than those predicted by NLO QCD by a factor of about 0.67 which indicates factorisation breaking. Contrary to naïve expectation, the value of this gap survival probability is the same in resolved and direct processes, showing no evidence for $x_{\gamma}$ dependence.

Several features of these new measurements reveal a complicated interplay of soft and hard phenomena which still represents a challenge for theoretical models of ep diffraction.

\section{References}

[1] J. Collins, Proof of factorisation for diffractive hard scattering, Phys. Rev. D 57 (1998) 3051 [Erratum: ibid. D 61 (1999) 019902]; [arXiv: 1006.0946 ].

[2] A. Affolder et al. [CDF Collaboration], Diffractive dijets with a leading antiproton in $\bar{p} p$ collisions at $\sqrt{s}=1800$ GeV, Phys. Rev. Lett. 84 (2000) 5043. 
[3] F.D. Aaron et al. [H1 Collaboration], Inclusive Measurement of Diffractive Deep-Inelastic Scattering at HERA, EPJ C72 (2012) 2074 [arXiv: 1203 . 4495].

[4] F.D. Aaron et al. [H1 Collaboration], Measurement of Dijet Production in Diffractive Deep-Inelastic Scattering with a Leading Proton at HERA, EPJ C72 (2012) 1970 [arXiv : 1111 . 0584].

[5] H1 Collaboration, Measurement of Diffractive Dijet Photoproduction with Leading Proton, H1prelim-13-011 (2013) [www-h1.desy.de/publications/htmlsplit/H1prelim-13-011.long.html]

[6] A. Aktas et al. [H1 Collaboration], Measurement and QCD analysis of the diffractive deep-inelastic scattering cross-section at HERA, EPJ C48 (2006) 715 [hep-ex/ 0606004 ].

[7] F.D. Aaron et al. [H1 Collaboration], Measurement of the cross section for diffractive deep-inelastic scattering with a leading proton at HERA, EPJ C71 (2011) 1578 [arXiv: 1010.1476 ].

[8] S. Chekanov et al. [ZEUS Collaboration], Deep inelastic scattering with leading protons or large rapidity gaps at HERA, Nucl. Phys. B816 (2009) 1 [arXiv: 0812 . 2003].

[9] C. Marquet, A unified description of diffractive deep inelastic scattering with saturation, Phys. Rev. D 76 (2007) 094017 [arXiv:0 0706.2682$].$

[10] A. Donnachie and P.V. Landshoff, Phys. Lett. B296 (1992) 227, e-Print Archive: hep-ph/9209205.

[11] Z. Nagy and Z. Trocsanyi, Multi-jet cross sections in deep inelastic scattering at next-to-leading order, Phys. Rev. Lett. 85 (2001) 082001 [hep-ph/ 0104315$].$

[12] H. Jung, Hard diffractive scattering in high-energy ep collisions and the Monte Carlo generator RAPGAP, Comput. Phys. Commun. 86 (1995) 147.

[13] S. Frixione, Z. Kunszt and A. Signer, Three jet cross-sections to next-to-leading order, Nucl. Phys. B467 (1996) 399 [hep-ph/9512328];

S. Frixione, A General approach to jet cross-sections in QCD, Nucl. Phys. B507 (1997) 295 [hep-ph/9706545]; See also http://www.ge.infn.it/ ridolfi/.

[14] F.D. Aaron et al. [H1 Collaboration], Diffractive Dijet Photoproduction in ep Collisions at HERA, EPJ C70 (2010) 15 [arXiv: 1006.0946$].$

[15] S. Chekanov et al. [ZEUS Collaboration], Diffractive photoproduction of dijets in ep collisions at HERA, EPJ C55 (2008) 177 [arXiv: 0710.1498 ]. 